\title{
El contexto urbano y las interacciones sociales: dualidad del espacio de actividades de sectores de ingresos altos y bajos en Concepción, Chile
}

Claudia García. Universidad de Concepción, Concepción, Chile.

Juan Antonio Carrasco. Universidad de Concepción, Concepción, Chile.

Carolina Rojas. Universidad de Concepción, Concepción, Chile.

RESUMEN | Este trabajo busca abordar la relación entre el contexto urbano y las interacciones entre las personas, identificando las dimensiones sociales y espaciales de esa relación, las que son enfocadas en dos niveles de análisis: el barrio, como base de los hogares de la muestra estudiada; y el individuo, en el contexto de sus redes sociales personales. Este análisis se hace a partir de casos de estudio individuales. La investigación se basa en un marco teórico interdisciplinar y en datos empíricos recogidos en Concepción, Chile. Los resultados sugieren la existencia de un espacio de actividades delimitado por los lugares donde los individuos desarrollan sus actividades sociales cotidianas, su entorno cercano. A ello se suma el espacio de movimiento que se conforma por la ruta o trayecto entre el hogar y trabajo de los individuos, y los lugares de interacción social con sus redes personales. Los análisis revelan la dualidad existente entre el espacio de actividades de sectores de ingresos altos y el de sectores de bajos ingresos, sugiriendo que la integración socioespacial y problemas concomitantes tiene ribetes más complejos y multidimensionales que los generalmente abordados en la literatura.

PALABRAS ClaVe $\mid$ redes, movilidad, desigualdad social.

ABSTRACT | The objective of this work is to study the relationship between the urban context and social interactions. The social and spatial dimensions involved in this relation were grouped into two levels of analysis: the neighborhood -as the stratum where household sample groups were taken - and the level of individuals in the context of their personal social networks. Individual case studies were carried-out as part of the analysis. The study is based in an interdisciplinary framework and empirical data from Concepcion, Chile. The results suggest the existence of a recreational space that is limited to the areas in which individuals establish their everyday social activities, and which generally represent their immediate surroundings. In addition to this immediate realm, there is also the space of movements formed by the path or route between the individual's home and workplace, and the places of social interaction with their personal net-works. The study reveals the duality between the recreational spaces of low-income groups and those of high-income groups, suggesting that issues of socio-spatial segregation touch upon wider, more complex and multidimensional aspects than those generally addressed in the literature.

KEY WORDS | networks, mobility, social inequality.

Recibido el 24 de agosto de 2012, aprobado el 25 de marzo de 2013

E-mail: Claudia García, cgarcia@udec.cl | Juan Antonio Carrasco, j.carrasco@udec.cl | Carolina Rojas, crojasq@udec.cl 


\section{Introducción}

Las interacciones sociales ocurren en un contexto social y urbano específico, gracias a las oportunidades y restricciones en la movilidad espacial de las personas involucradas. Motivados por el importante vacío en la literatura al respecto y el creciente interés en las perspectivas de movilidad en torno a las interacciones sociales, el objetivo del siguiente trabajo es estudiar la relación entre el contexto urbano y las interacciones sociales para barrios de ingresos altos y bajos de la ciudad de Concepción. Con tal fin, el estudio se concentra en las redes sociales personales de individuos de ingresos altos y bajos, pertenecientes a barrios con distintos niveles de accesibilidad al centro de comercio y servicios de la ciudad.

El artículo identifica factores que relacionan la dimensión social con la dimensión espacial de las actividades sociales de individuos, a partir de las redes sociales personales subyacentes y de las características del contexto urbano en que habitan (hogares y trayectos de viajes). De esta forma, la dimensión social se estudia mediante las características de las personas con las cuales ciertos individuos interactúan y realizan actividades sociales en el espacio. En la dimensión espacial se indaga por la localización geográfica donde ocurren las interacciones y actividades sociales, incluyendo características de su contexto urbano, las que pueden tener incidencia sobre la capacidad de los individuos de participar en dichas actividades.

El viaje y el lugar de encuentro construyen el escenario donde los individuos se relacionan entre sí y con su entorno, por medio de las actividades sociales. La experiencia vivida en este escenario le confiere un significado que emerge del contexto social, y se sustenta en las relaciones sociales entre los actores. Estas relaciones sociales, construidas a partir de redes sociales, se manifiestan temporal y espacialmente también como una red, que puede ser geográficamente representada como un espacio de apropiación o de actividades.

El artículo propone un enfoque de Análisis de Redes Sociales (ARs) para identificar el contexto social en el que se desarrollan las interacciones entre los individuos, a partir de la comprensión de las características de los contactos con quienes los individuos interactúan de forma más frecuente, y con quienes realizan actividades sociales. El vínculo de las redes sociales con el contexto urbano se establece a partir de la localización de los lugares de interacción y actividades, configurando así el espacio de actividades sociales de cada individuo, compuesto tanto por los lugares de encuentro (nodos), como por el "espacio de movimiento" generado por el desplazamiento desde un lugar a otro (ruta de viaje) para acceder a una actividad social dada (Grannis, 2009; Netto \& Krafta, 2001).

Los principales aportes del trabajo son una aproximación teórica basada en tres elementos: las interacciones sociales, el contexto urbano y el comportamiento del transporte. Estos tres elementos son estudiados empíricamente para establecer los nexos apropiados entre aspectos sociales y espaciales, considerando una serie de indicadores cuantitativos (Boarnet \& Crane, 2001; Cervero, 2003; Crane, 2000; Ewing \& Cervero, 2010; Ewing, Handy, Brownson, Clemente \& Winston, 2006; Handy, Cao \& Mokhtarian, 2005; Kitamura \& Mokhtarian, 
1997). Este análisis permite revelar la diferencia en el nivel de accesibilidad de individuos de distintos ingresos a sus contactos sociales, revelando la dualidad existente en sus espacios de actividades sociales.

\section{Marco teórico}

Las interacciones sociales en el contexto de las redes sociales

Wellman (2001) define "comunidad" como las redes de lazos interpersonales que proveen sociabilidad, apoyo, información, sentido de pertenencia e identidad social. En una sociedad de redes, las interacciones involucran distintos actores en distintos niveles y los vínculos conectan muchas redes. En las redes sociales, los actores y sus acciones son interdependientes entre sí, con lazos que sirven para transferencias de recursos materiales y no materiales, y que actúan como una potente herramienta en la construcción del capital social (Lin, 2005).

El desarrollo de las Tecnologías de Información y Comunicación (TIC) ha posibilitado últimamente que las redes sociales estén aún más extendidas en su distribución espacial que en el pasado, de modo que la existencia y el alcance de sus lazos ya no enfrentan barreras geográficas ni se ven restringidas por la distancia física entre sus actores. Sin embargo, la distancia entre actores sigue siendo relevante

(Mok, Wellman \& Carrasco, 2010), especialmente en redes sociales personales de individuos de escasos recursos, los que presentan una notable característica de "localismo", como es el caso de Brasil (Holanda, 2000; Marques, Moya \& Zoppi, 2008).

\section{El contexto urbano}

El contexto urbano se ha analizado tradicionalmente con variables como densidad, diversidad y diseño de la trama vial (Boarnet \& Crane, 2001; Cervero, 2003; Crane, 2000; Ewing \& Cervero, 2010; Ewing et al., 2006; Handy et al., 2005; Kitamura \& Mokhtarian, 1997). Está definido por un espacio configurado, que puede ser más o menos continuo, característica que incide en facilitar la fricción entre personas (Grannis, 1998; 2009). Este espacio configurado puede condicionar el movimiento de los individuos en los distintos lugares de la ciudad que ocupan (Hillier \& Hanson, 1984; Hillier, 1996; Netto \& Krafta, 2001), y constituir el escenario donde se desarrollan actividades de interacción social entre los individuos (Fan \& Khattak, 2009).

Grannis (2009) amplía esta perspectiva, revelando que la red de calles peatonales también influye en la forma en que la población se organiza espacialmente de acuerdo con características como su raza, para el caso de California, Estados Unidos. Es decir, la continuidad del espacio urbano de las calles peatonales actuaría promoviendo la interacción social; sería relevante en la elección de los lugares de residencia por un determinado grupo social, permitiría una mayor fricción de contactos entre vecinos y aumentaría la probabilidad de interacción pasiva entre individuos de características sociodemográficas semejantes. En esta misma línea, la teoría de la Space Syntax (Hillier \& Hanson, 1984; Hillier, 1996) postula una relación simbiótica entre la configuración espacial y las actividades humanas, suponiendo que el espacio -cuya forma encierra una geometría y una topología- está 
configurado por la organización y vinculación entre distintos subespacios continuos que establecen un determinado patrón de conexiones. De esta forma, la noción de una red socioespacial sirve para entender la configuración del sistema social y urbano de actividades como un constructo social correlacionado (Netto \& Krafta, 2001). El movimiento que se desarrolla en función de la red de calles en la ciudad, conformada por un espacio configurado medible y que Marshall (2005) denomina como rutas, permite establecer un patrón de movimiento. Este, a su vez, puede describir el comportamiento de los individuos en las interacciones y actividades que se desarrollan en esos espacios continuos, y los modos de transporte utilizados para moverse en él.

\section{Comportamiento del transporte y el contexto urbano}

Los estudios de comportamiento de transporte han evolucionado en los últimos cincuenta años, pasando por tres enfoques (Jones, 2009). El primero se basa en los viajes; la segunda perspectiva se centra en las actividades, asumiendo que los viajes son una actividad derivada de otros quehaceres (trabajo, estudio, compras, etcétera). El tercer enfoque se centra en las actitudes personales, bajo el supuesto de que el comportamiento no solo se relaciona con aspectos objetivos de la oferta de transporte, sino también con atributos más subjetivos, relacionados con las interacciones sociales de los individuos que emergen en un contexto social y urbano específico (Ronald \& Arentze, 2009; Axhausen, 2006; Carrasco \& Miller, 2006).

La localización residencial y su relación con la distancia respecto de otras actividades del hogar es un factor que también ha estado presente en algunos estudios, en una mirada de macroescala, en términos de política de planificación y ordenamiento territorial (Næss, 2005). Además, este atributo es utilizado para establecer parámetros para la definición de índices de accesibilidad (Kitamura, Akiyama, Yamamoto \& Golob, 2001).

CUADRo 1 | Matriz dimensiones de los atributos contexto social y urbano

\begin{tabular}{|c|c|c|c|c|}
\hline \multirow{2}{*}{ DIMENSIÓN } & \multirow{2}{*}{$\begin{array}{c}\text { ATRIBUTOS CONTEXTO SOCIAL } \\
\text { Y URBANO }\end{array}$} & \multicolumn{3}{|c|}{ ENFOQUE } \\
\hline & & VIAJES & ACTIVIDADES & ACTITUDES \\
\hline \multirow{9}{*}{ ESPACIAL } & Densidad & $x$ & $x$ & \\
\hline & Diversidad & $\mathrm{x}$ & & \\
\hline & Diseño & $\mathrm{x}$ & & \\
\hline & Estética & & & $\mathrm{x}$ \\
\hline & Distancia commuting & & & $\mathrm{x}$ \\
\hline & Distancia centros de servicio & & $\mathrm{x}$ & $\mathrm{x}$ \\
\hline & Facilidad transporte & & $\mathrm{x}$ & $\mathrm{x}$ \\
\hline & Lugar de interacción & & $\mathrm{x}$ & \\
\hline & Existencia de escuelas & & & $\mathrm{x}$ \\
\hline
\end{tabular}

(continúa) 
(continuación)

\begin{tabular}{|c|c|c|c|c|}
\hline \multirow{2}{*}{ DIMENSIÓN } & \multirow{2}{*}{$\begin{array}{c}\text { ATRIBUTOS CONTEXTO SOCIAL } \\
\text { Y URBANO }\end{array}$} & \multicolumn{3}{|c|}{ ENFOQUE } \\
\hline & & VIAJES & ACTIVIDADES & ACTITUDES \\
\hline \multirow{13}{*}{ SOCIAL } & Capacidad de interacción con vecinos & & & $\mathrm{x}$ \\
\hline & Seguridad & & & $\mathrm{x}$ \\
\hline & Valor residencia & & & $\mathrm{x}$ \\
\hline & Nivel de ingreso & & $\mathrm{x}$ & $\mathrm{x}$ \\
\hline & Tamaño hogar & & $\mathrm{x}$ & $\mathrm{x}$ \\
\hline & Nivel educación & & $\mathrm{x}$ & $\mathrm{x}$ \\
\hline & Ocupación & & $\mathrm{x}$ & $\mathrm{x}$ \\
\hline & Género & & $\mathrm{x}$ & $\mathrm{x}$ \\
\hline & Edad & & $\mathrm{x}$ & $\mathrm{x}$ \\
\hline & Tenencia auto particular & & $\mathrm{x}$ & $\mathrm{x}$ \\
\hline & Lugar de trabajo & & $\mathrm{x}$ & $\mathrm{x}$ \\
\hline & $\mathrm{N}^{\circ}$ de familias en la vivienda & & $\mathrm{x}$ & $\mathrm{x}$ \\
\hline & Ciclo de vida & & $\mathrm{x}$ & $\mathrm{x}$ \\
\hline \multirow{3}{*}{ TEMPORAL } & Tiempo libre & & $\mathrm{x}$ & $\mathrm{x}$ \\
\hline & Frecuencia de interacción con vecinos & & $\mathrm{x}$ & \\
\hline & Horario funcionamiento actividades & & $\mathrm{x}$ & \\
\hline
\end{tabular}

FUENTE ELABORACIÓN PROPIA BASADA EN REVISIÓN BIBLIOGRÁFICA

El Cuadro 1 sintetiza algunos de los elementos centrales presentes en la literatura, relacionando las dimensiones espacial, social y temporal, con los enfoques basados en los viajes, las actividades y las actitudes personales. Estas dimensiones definen las variables posibles de medir en cada ámbito y los enfoques (viajes, actividades, actitudes) en que se enmarca el análisis de la relación del contexto urbano con el transporte.

\section{Vínculos entre las dimensiones espacial y social}

El contexto urbano constituye un soporte relevante en la conformación y mantenimiento de las comunidades individuales (Grannis, 2009), sirviendo de catalizador de actividades e interacción social; por ejemplo, parques o centros comerciales cercanos a los lugares de residencia facilitarían la actividad social conjunta en el contexto familiar (Fan \& Khattak, 2009). Aunque la interacción y la actividad social implican un desplazamiento desde un lugar a otro, no solo interesa el barrio de residencia de las personas estudiadas, sino también el contexto urbano constituido por el "espacio de movimiento" que conforma la ruta de viaje para acceder a una actividad social dada. Este espacio tiene como soporte la red de calles donde se localizan las residencias de los individuos (red de calles del barrio), junto con la red construida a partir de los desplazamientos de los individuos, como producto de las interacciones y actividades sociales entre los integrantes de su red social (red de rutas de viaje). Esta perspectiva permite establecer un vínculo o superposición entre 
la estructura de las redes personales y la red vial (Marshall, 2005), configurada a partir de las rutas de viaje, producto de las interacciones sociales de los individuos.

Recientes investigaciones han buscado entender el rol del contexto social de los individuos en la espacialidad y temporalidad de sus actividades sociales y consecuente movilidad cotidiana, a partir de un enfoque de redes sociales (Carrasco \& Miller, 2006; 2009; Wellman, 2001). De esta forma, el Análisis de Redes Sociales (ARS) permite vincular los atributos personales y la composición de la red social de individuos específicos, con el contexto urbano (McCarty, 2002; McPherson, SmithLovin \& Cook, 2001; Wellman, 1998). A tal efecto, estudia, por ejemplo, la existencia de ciertos tipos de equipamientos en las cercanías de las residencias o lugares de trabajo, que pueden favorecer la interacción social entre miembros de una red personal (Fan \& Khattak, 2009; Ronald \& Arentze, 2009). Más en general, el rol del contexto urbano en las interacciones sociales puede analizarse acorde a su tipología (ambientes cerrados o abiertos, públicos o privados) y considerando si reúne o no condiciones que favorecen esas interacciones sociales (Fan \& Khattak, 2009).

El Cuadro 2 sintetiza las dimensiones necesarias de considerar para integrar en el análisis los diferentes aspectos que reviste este problema multidisciplinar y multiescalar. Tales dimensiones son la espacial y la social.

CUADro 2 Dimensiones y niveles de análisis del estudio

\begin{tabular}{|c|c|c|}
\hline \multirow{2}{*}{ DIMENSIÓN } & \multicolumn{2}{|c|}{ NIVEL ANÁLISIS } \\
\hline & BARRIO & INDIVIDUO \\
\hline \multirow{9}{*}{ ESPACIAL } & 1. Características físico-arquitectónicas & 1. Lugares de interacción \\
\hline & Morfología urbana & Tipos \\
\hline & Calidad urbanización & Localización \\
\hline & Densidad habitacional & \\
\hline & Nivel ocupación suelo & \\
\hline & Usos de suelo & 2. Rutas de viaje \\
\hline & Nivel equipamientos & Modos \\
\hline & Estructura vialidad & Estructura de rutas \\
\hline & Cobertura transporte & \\
\hline \multirow{10}{*}{ SOCIAL } & 1. Características socioeconómicas & 1. Estructura de la red social \\
\hline & Nivel promedio ingreso & Homofilia \\
\hline & Ciclo de vida & Tamańo \\
\hline & Género & Grado \\
\hline & Nivel de educación & Intermediación \\
\hline & Ocupación & Cercanía \\
\hline & 2. Características de las redes sociales & Densidad \\
\hline & & Aislados \\
\hline & & Componentes \\
\hline & & Cliques \\
\hline
\end{tabular}


En el caso de la dimensión espacial se distinguen dos niveles de análisis: el barrio y el individuo. Respecto del barrio, se analizan aspectos identificados tanto en la literatura tradicional de la investigación de transporte (Boarnet \& Crane, 2001; Cervero, 2003; Crane, 2000; Ewing \& Cervero, 2010; Ewing et al., 2006; Handy et al., 2005; Kitamura \& Mokhtarian, 1997) como en la literatura de morfología urbana (Panerai, 2006; Solá-Morales, 1996). Además se emplean otros atributos relevantes, como la densidad habitacional, nivel de ocupación del suelo, actividades, nivel de equipamientos, estructura de la vialidad y cobertura de transporte, entre otros, estableciendo un conjunto de indicadores que permiten comparar sectores de la ciudad con distintas características. Respecto del individuo se deben incorporar las características de sus lugares de interacción y actividades sociales, como su tipología y localización. Además, interesa conocer las rutas de viaje que se generan producto de las actividades sociales entre los individuos y el modo utilizado en estos viajes.

En el caso de la dimensión social, a escala de barrio se definen aspectos socioeconómicos que inciden en las características de las redes sociales, como ciclo de vida, nivel educacional, ocupación, género y nivel de ingreso, que tienen potencial relevancia en la caracterización general del grupo de estudio en cuanto a dar luces respecto de la localización de las actividades sociales. Por otro lado, la estructura de la red social personal establece otros aspectos que potencialmente inciden en la interacción social, como la homofilia (McPherson, Smith-Lovin \& Cook, 2001) y estructura de los contactos (Marques et al., 2008; Grande, 2007).

\section{Marco metodológico}

\section{Descripción de los datos}

Los datos utilizados en este trabajo fueron obtenidos por la investigación "Sociedad, transporte y comunicación: redes y comunidades del Gran Concepción”, realizada entre julio del 2008 y enero de 2009 a 241 encuestados de cuatro muestras de individuos de la comuna de Concepción, Chile, que captura las principales características socioeconómicas y de movilidad de los participantes (Carrasco, Bustos \& CidAguayo, 2013). Los encuestados provienen de dos sectores de bajos ingresos: Agüita de la Perdiz [AP] $(n=60)$, y Santa Sabina [ss] $(n=60)$; y dos a sectores de ingresos altos: Barrio Universitario/La Virgen [BU/Lv] ( $\mathrm{n}=61)$, y Lomas de San Sebastián/ Lomas de San Andrés [LSS/LSA] ( $\mathrm{n}=60)$. La elección de dos de estos barrios (AP y BU/LV) apunta a su cercanía con el centro de servicios del Área Metropolitana de Concepción (en adelante AMc), lo que implica facilidades de acceso a la red de transporte, salud, educación, comercio y trabajo. Esta situación fue resultado de las primeras transformaciones del AMC hacia un proceso de metropolización, impulsado a partir de la implantación del sector industrial en la década de 1950, coincidente con las tendencias migratorias desde los sectores rurales a los centros urbanos (Pérez \& Salinas, 2009). Los otros dos sectores (ss y LSS/LSA) se encuentran localizados en una condición de mayor lejanía al centro, entre 4 y 6 kilómetros de distancia, y cuentan con menor acceso a servicios en general. El alcance geográfico de este trabajo estuvo delimitado por la distribución espacial de las redes sociales personales localizadas dentro de los límites del AMC. 


\section{Unidades de análisis}

El análisis se realizó a escala de barrio e individuo, comparando la dimensión espacial con aspectos de la morfología urbana (contexto urbano), como los sistemas vial, parcelario y edificatorio, y cobertura de transporte y oferta de actividades en el ámbito local. A nivel individual se analizaron los lugares de interacción más frecuente y las rutas de viaje.

La dimensión social a escala de barrio incluye dos aspectos: las características socioeconómicas de los grupos muestrales, como nivel promedio de ingreso, nivel de educación, género y ocupación; y aspectos referentes a la composición del hogar y con quiénes se convive en él, junto con características de las redes sociales. En lo individual se analizaron indicadores de la estructura de la red social personal de los entrevistados, a partir de indicadores desarrollados por el ARs (McCarty, 2002). En la individualización de los sectores de ingresos altos cada barrio se subdividió en dos áreas, por ser perceptualmente distintas, aunque cumpliendo con la condición de distancia de cercanía con el centro urbano, como es el caso de LV y BU, y de una cierta mayor lejanía en el caso de LSS y sA.

\section{Descripción de los barrios}

\section{Agüita de la Perdiz (ap)}

El asentamiento que conforma el barrio es producto de una toma de terreno que data de 1958, la más antigua del Gran Concepción (Agurto, 2002), resultado de procesos migratorios regionales. El barrio se ha desarrollado y extendido sobre las faldas del cerro Caracol, condición geográfica que le imprime al sector características morfológicas muy particulares y que incide directamente tanto en la dificultad de acceso a algunas viviendas como en la implementación de obras de urbanización todavía precarias en algunos sectores del barrio.

Parte de estas características, sumadas a los rasgos de cohesión comunitaria entre los pobladores - una estrategia de sobrevivencia y superación de adversidades frente a las condiciones de vida precarias y de riesgo social que los caracterizan-, definen el barrio como una suerte de enclave al interior del contexto en que se desarrolla. Adicionalmente, cabe resaltar su cercanía con el centro de Concepción, donde está localizada la mayor oferta de comercio, servicios y equipamientos de salud y educación; entre otros, la Universidad de Concepción y el Hospital Regional Guillermo Grant Benavente.

\section{La Virgen (LV) y Barrio Universitario (BU)}

Los barrios LV y BU surgieron gracias a la demanda de terrenos destinados a la construcción de viviendas para un creciente número de profesionales que llegaron a la zona a raíz de la instalación del sector industrial. Pese al origen común con AP, ambos sectores tienen características morfológicas sensiblemente distintas, ya que LV se desarrolló en las faldas del cerro del mismo nombre hacia el sector nororiente del casco urbano central, y el вu lo hizo a partir de la extensión del tejido urbano del casco central, que gradualmente se fue adaptando orgánicamente a las faldas del cerro Caracol. Ambos sectores se encuentran relativamente cercanos, con alrededor 
de 600 metros entre sus bordes de acceso. La distancia entre estos sectores y la plaza de Armas de la comuna es de $1,5 \mathrm{~km}$, lo que les confiere un alto grado de accesibilidad a equipamientos de comercio, servicio, salud y educación, además de contar con una buena oferta de transporte público en calles que bordean el sector.

\section{Santa Sabina (SS)}

Santa Sabina, un caso característico de asentamiento urbano ocurrido a partir de la ocupación de terrenos agrícolas que conformaban el sector periférico de la ciudad, se generó mediante seis cooperativas, creadas en 1969. El asentamiento se desarrolló en un territorio de topografía irregular, cuyo paisaje se desenvuelve por colinas y cerros. La distancia que lo separa del principal centro de comercio y de servicios de la comuna de la ciudad es de aproximadamente $4,5 \mathrm{~km}$, medidos hasta la plaza de Armas de Concepción. El sector cuenta con una buena oferta de transporte público, con diversas líneas de taxibuses y taxis colectivos hacia el centro de la ciudad.

\section{Lomas de San Sebastián (LSS) y Lomas de San Andrés (LSA)}

El sector de LSA fue el primero en desarrollarse, a comienzos de los ańos ochenta, a partir de la actuación de agentes inmobiliarios y la urbanización de terrenos de bajo costo -aunque de alto valor de construcción, por la necesidad de contención de los terrenos, debido a las características topográficas del lugar-. La venta de los terrenos estaba dirigida principalmente a la autoconstrucción por sectores de ingresos alto y medio-alto, con viviendas en los cerros y colinas cuya ventaja era su localización cercana a infraestructura vial de conexión hacia distintos sectores de la intercomuna.

El sector de Lss, en cambio, es un área de vegas y plano, cuyo desarrollo se inició a partir de los ańos noventa. Cabe seńalar que los proyectos inmobiliarios que le dieron origen también establecieron un nuevo concepto de vivienda urbana para la época: los conjuntos habitacionales en condominios cerrados. Los conjuntos que lo conforman son ejemplo de una forma de crecimiento caracterizada por operaciones unitarias, en general bajo el impulso de agentes inmobiliarios, que insertan a un grupo social (estrato socioeconómico alto o medio-alto) en una suerte de isla dentro de o aledaña a conjuntos habitacionales de familias de menores ingresos, constituidos por viviendas sociales o de autoconstrucción (Pérez \& Salinas, 2009).

La distancia entre estos dos barrios, LSS y LSA, es de aproximadamente $1,5 \mathrm{~km}$, y distan alrededor de $4 \mathrm{~km}$ y $6 \mathrm{~km}$, respectivamente, del centro comercial de Concepción.

La oferta de transporte público es inferior a la encontrada en los demás sectores, destacándose los taxis colectivos como principal modo. De acuerdo con las entrevistas, se percibe que la relación entre vecinos es amistosa, pero distante, con muy pocos lazos emocionales de importancia.

Cabe seńalar que el sector El Trébol, cuya cercanía a los sectores de LSA, Lss y ss está entre 1 y $3 \mathrm{~km}$, por su condición estratégica dentro del territorio y con buena infraestructura vial, ha sido favorecido por la instalación de actividades y servicios a partir de grandes equipamientos de comercio y nuevos espacios de encuentro y recreación, dirigidos especialmente hacia sectores de ingresos medios y altos. Lo anterior ha generado una nueva centralidad en paralelo al centro de servicios 
tradicional del AMC, fenómeno que muestra una tendencia de crecimiento metropolitano con una inclinación hacia un modelo más disperso, de mediana complejidad, favorecedor del uso del transporte privado, con formas más complejas y en dirección a las periferias y cerros (Rojas, Opazo \& Jaque, 2009).

\section{Análisis de la dimensión espacial}

El contexto urbano fue abordado desde dos niveles de escala. El primero está relacionado con la localización de los grupos muestrales, filtro para la caracterización socioeconómica de los individuos en cada barrio y la caracterización de este, entendido como una unidad perceptual en la cual están insertos los hogares de los individuos encuestados. El segundo nivel se relaciona con el contexto urbano. Implica una vinculación del entorno inmediato con los lugares donde se da una interacción social más frecuente. En esta escala se establece una relación entre el sector en estudio y la estructura de red de los recorridos, conformada por las rutas o trayectos supuestos de viaje para la realización de las interacciones sociales encontradas, ámbito que configura el espacio de actividad individual.

La morfología urbana se analizó con la comparación entre las características físicas del tejido urbano de cada sector, como la estructura viaria, parcelación de suelo y edificación. Se aplicó adicionalmente el enfoque de la teoría de Space Syntax, usando la metodología planteada por Marshall (2005) para el análisis de la estructura de la red vial de cada sector, el que permitió establecer ciertas similitudes y diferencias entre ellos y medir sus grados de integración local y global con el resto de la ciudad.

A nivel individual, el análisis del contexto urbano se centró en la estructura del espacio de actividades (Axhausen, 2006; Schonfelder \& Axhausen, 2003), y fue realizado a partir de algunos casos de estudio en cada barrio. El espacio de actividades consideró tres niveles de contactos, que conforman las rutas entre orígenes y destinos: (i) hogar de participantes y hogar de contactos; (ii) hogar de participantes y lugar de interacción frecuente; y (iii) hogar de participantes y lugar de actividad social. Además de lo anterior, se incorporó información acerca del lugar de trabajo del participante. Cabe notar que los trayectos entre un punto y otro por cada nivel asumen la menor distancia posible en la red vial existente. Para estos análisis se utilizaron Sistemas de Información Geográfica para localizar espacialmente la red social personal de los individuos ubicados al interior del AMc.

\section{Análisis de la dimensión social}

El primer aspecto analizado en la dimensión social es la caracterización socioeconómica de los grupos en estudio. Variables como sexo, edad, nivel de ingreso, nivel de educación y estado de ocupación fueron consideradas para detectar presencia de homofilia (similitud en características) en los individuos y en las redes personales de los grupos muestrales. Otras variables, como los ańos de ocupación de la vivienda y la propiedad legal de ella, fueron considerados para medir el grado de arraigo de cada grupo estudiado en el sector donde vive, así como la incidencia de este factor en los vínculos generados entre vecinos y amigos al interior del barrio.

En materia de los datos referidos a la estructura de las redes sociales se consideraron principalmente valores promedio de densidad (indicador de lazos existentes en 
la red), centralidad (indicador del nivel de actividad de la red), cercanía (medida de independencia del control de otros integrantes de la red), e intermediación (medida de control de información); todo lo anterior con el objetivo de medir características de cohesión entre los distintos grupos muestrales. Además, se utilizaron otros indicadores para medir el grado de compartimentalización y subgrupos de las redes: componentes (grupo de participantes de la red que están conectados entre sí directa o indirectamente) y cliques (grupos donde todos los participantes se conocen entre sí), basados en metodologías de ARs (McCarty, 2002; McPherson et al., 2001).

A nivel individual se emplearon casos de estudio en cada sector para examinar la red personal de los individuos. A partir del enfoque de ARs, se realizó un análisis de la estructura de esas redes personales, identificando qué relación con el individuo tienen sus contactos sociales, y con quiénes realizan actividades sociales, localizándolos en el espacio de actividades del participante (Figura 1). La visualización de la estructura de la red personal representada topológicamente en un grafo permitió vincularla con el contexto urbano por medio de la superposición de esta red de personas con la red que conforma el "espacio de movimiento" o trayectos entre el participante y los lugares de interacción más frecuente con sus contactos (Figura 2).

FIGURA 1 | Esquema de red social personal
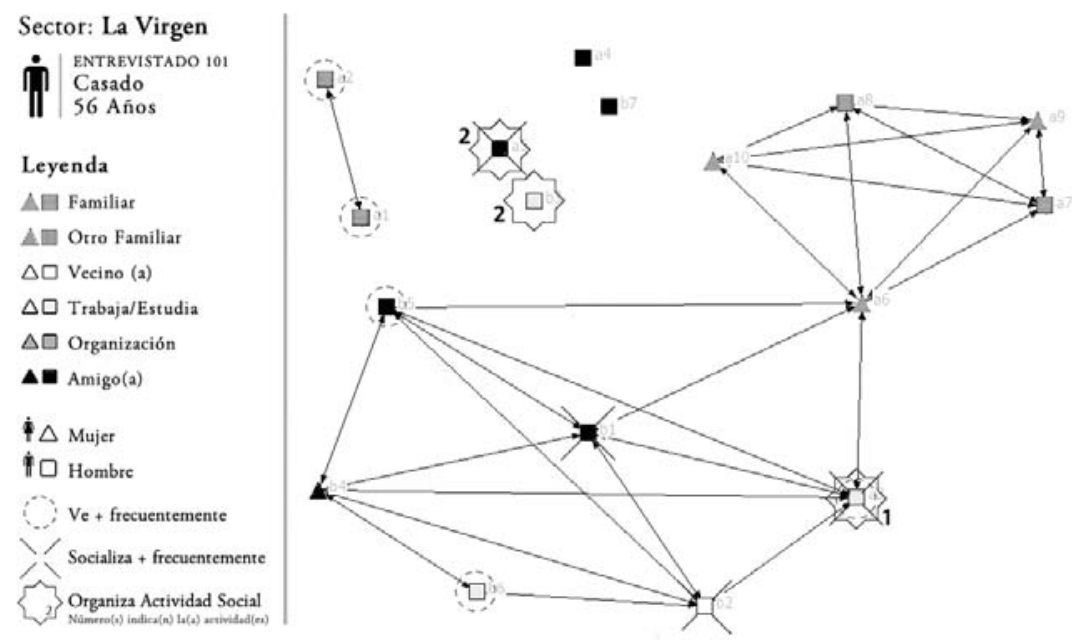

FUENTE ELABORACIÓN PROPIA

De esta manera se pudo visualizar espacialmente el vínculo entre el individuo participante y cada uno de sus contactos, inserto en el contexto urbano, estudiando la distancia entre ellos a partir de la red vial.

La red de rutas tiene una estructura de arcos y nodos, generados por la red vial en la cual está inserta, lo que permite el análisis de su estructura topológica por el enfoque metodológico de Marshall (2005). Se forma a partir de dos componentes: (i) la red personal social del participante, considerando su hogar y lugar de trabajo como puntos iniciales de los trayectos, en relación con la localización espacial de los hogares de sus contactos sociales; y (ii) a los lugares más frecuentes de interacción 
social con ellos. Lo anterior permite configurar el espacio de actividades estudiado en relación con las características principales del individuo, los lugares de interacción (diferenciando tipo, densidad y diversidad de usos), y el contexto urbano donde están insertas esas interacciones sociales.

\section{FIGURA 2 Espacio de actividades}

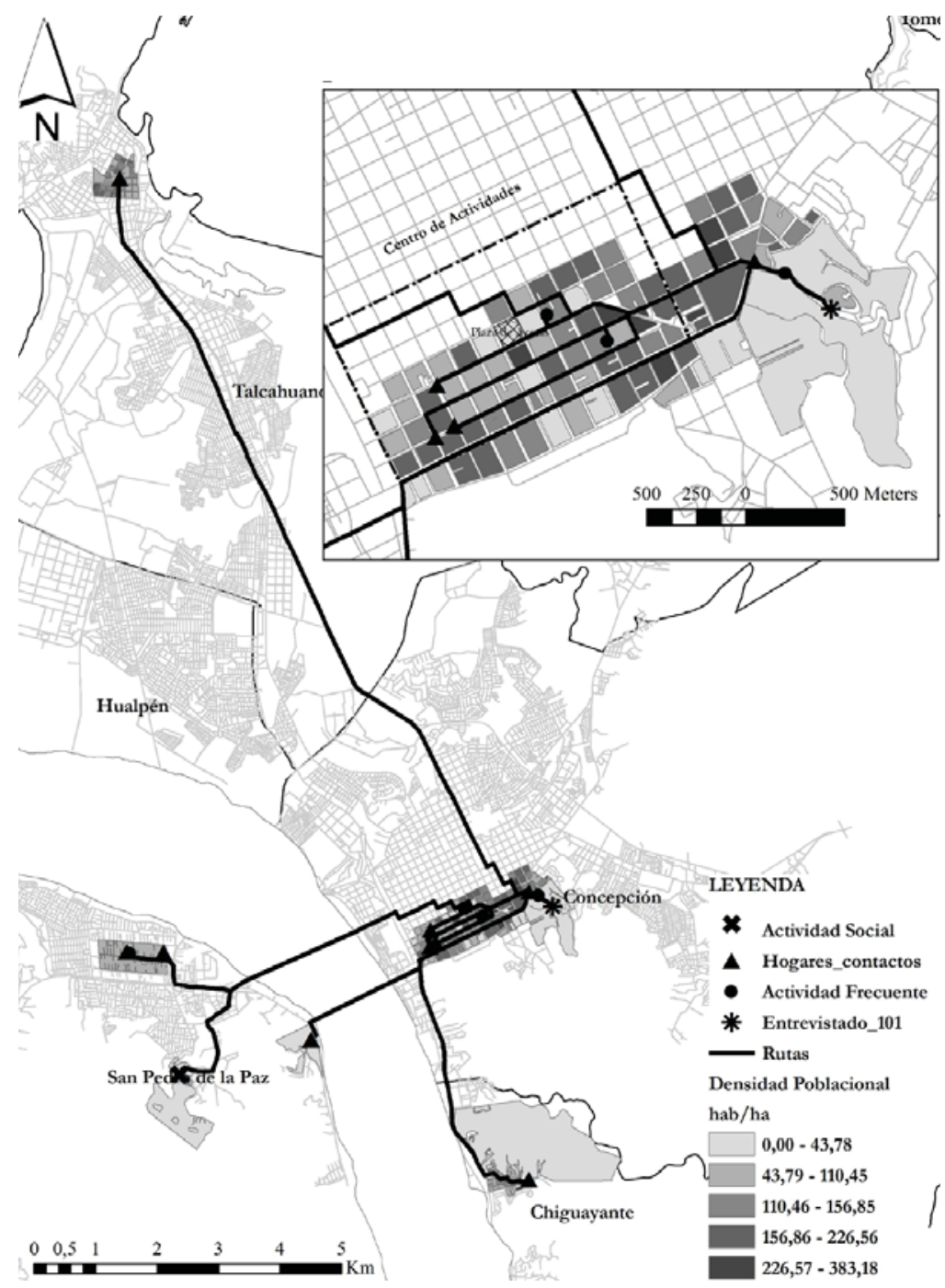

FUENTE ELABORACIÓN PROPIA

\section{Análisis de los resultados}

\section{Morfología urbana}

Los sectores en estudio se desarrollaron con una forma de crecimiento urbano propia de áreas suburbanas. Incluso el sector del BU -que a primera vista podría entenderse como una extensión de la trama regular del centro urbano- finalmente termina por presentar una estructura de red mixta, compuesta por muchas rutas 
de vías tributarias, muy características de la configuración suburbana discutida por Solá-Morales (1996) y otros autores (Marshall, 2005; Pérez \& Salinas, 2009; Stead $\&$ Marshall, 2001), como se aprecia en la Figura 3.

FIGURA 3 | Comparación tejido urbano
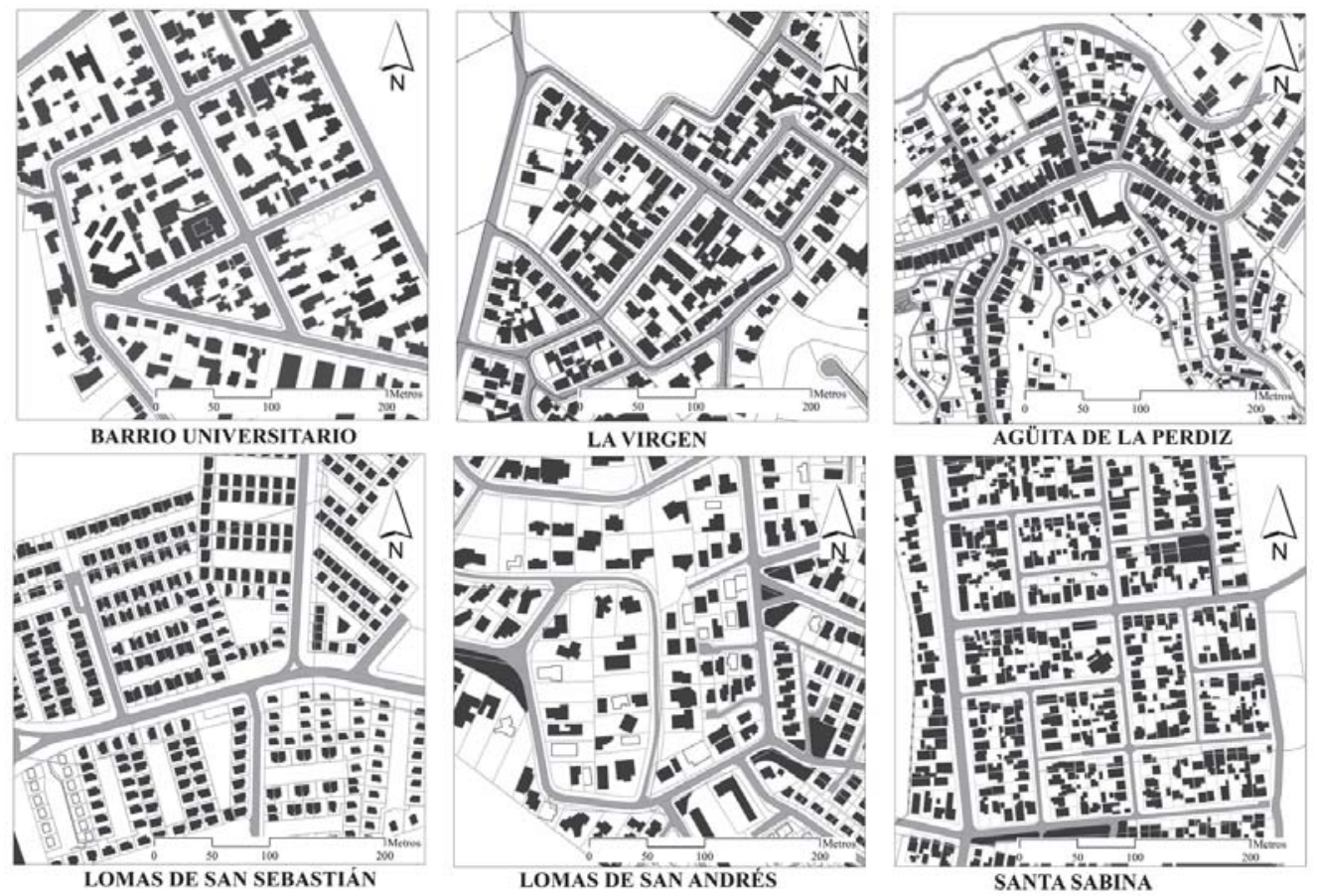

FUENTE ELABORACIÓN PROPIA A PARTIR DE FOTOS Y VISITAS A TERRENO

Los resultados permiten vislumbrar que los sistemas viales de los dos sectores de ingresos bajos ofrecen una mejor condición de conectividad interna que sus contrapartes. Esta condición morfológica permitiría una fricción más constante entre los integrantes de las redes personales barriales.

El Cuadro 3 muestra los valores de continuidad, conectividad y profundidad relativa, que miden propiedades de la red y permiten la comparación entre los sistemas viales de cada sector. Las medidas de continuidad relativa se refieren a la integración interna entre los arcos que conforman cada ruta, mientras que la profundidad indica la posición de conectividad relativa de una ruta en función del contexto de la red en que está inserta.

La comparación entre los valores del Cuadro 3 muestra un alto nivel de integración interna en todos los casos (dado por el valor de la continuidad), siendo el BU, AP y ss los que poseen valores más altos. Esta condición favorece el uso de la caminata como modo de transporte, y es coherente con el resultado de modo elegido para visitar amigos y familiares en los sectores de AP y ss, como se puede apreciar en el Gráfico 1. 
CUADro 3 | Propiedades de la red según Marshall

\begin{tabular}{|l|r|r|r|r|r|r|r|c|}
\hline \multirow{2}{*}{\multicolumn{1}{|c|}{ SECTOR }} & \multicolumn{7}{|c|}{ PROPIEDADES DE LA RED } \\
\cline { 2 - 11 } & $\mathbf{1}$ & $\mathbf{c}$ & $\mathbf{d}$ & $\begin{array}{c}\text { Cantidad } \\
\text { tipos }\end{array}$ & Suma (s) & L & C & D \\
\hline Agüita la Perdiz & 73 & 129 & 127 & 19 & 329 & 0,39 & 0,22 & 0,39 \\
\hline B. Universitario & 41 & 64 & 51 & 16 & 156 & 0,41 & 0,26 & 0,33 \\
\hline La Virgen & 50 & 71 & 94 & 18 & 215 & 0,33 & 0,23 & 0,44 \\
\hline Santa Sabina & 214 & 256 & 200 & 33 & 670 & 0,38 & 0,32 & 0,3 \\
\hline Lomas S. Sebastián & 24 & 25 & 31 & 9 & 80 & 0,31 & 0,3 & 0,39 \\
\hline Lomas S. Andrés & 183 & 215 & 246 & 31 & 645 & 0,33 & 0,28 & 0,38 \\
\hline
\end{tabular}

$\mathrm{l}$ = continuidad; $\mathrm{c}=$ conectividad; $\mathrm{d}=$ profundidad

$\mathrm{L}=$ continuidad relativa; $\mathrm{C}=$ conectividad relativa; $\mathrm{D}=$ profundidad relativa

FUENTE ELABORACIÓN PROPIA

\section{GRÁFICO 1 | Modos utilizados para visitar amigos y familiares}

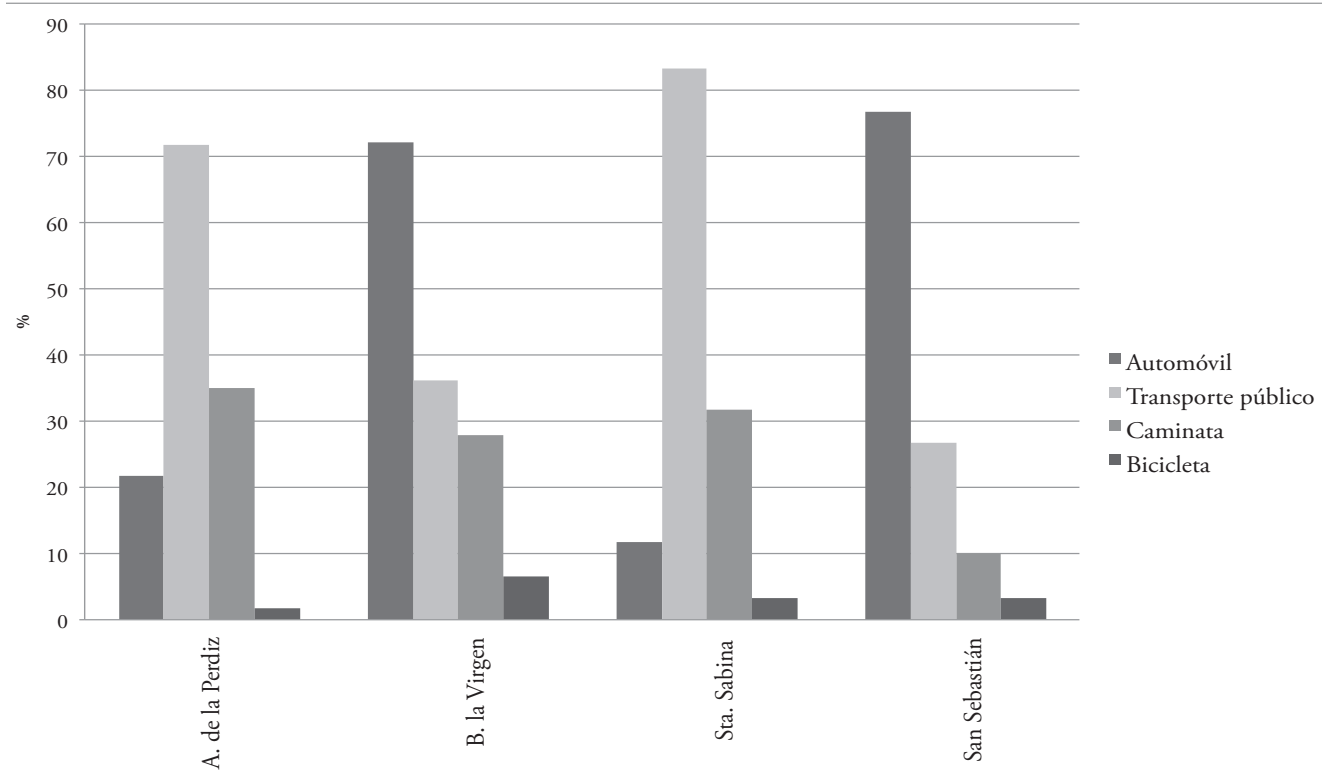

FUENTE ELABORACIÓN PROPIA BASADA EN DATOS RECOLECTADOS

La configuración de los distintos tejidos urbanos de los barrios se relaciona con el grupo socioeconómico que lo construye, alcance coherente con discusiones previas en la literatura respecto del proceso de transformación de la ciudad (Pérez \& Salinas, 2009; Rojas et al., 2009). Este resultado es también congruente con la literatura de transporte, que discute el rol de la demanda de viajes (Boarnet \& Crane, 2001; Crane, 2000; Ewing \& Cervero, 2010) y la elección residencial (Mokhtarian \& Cao, 2008; Cao, Mokhtarian \& Handy, 2007; Handy et al., 2005) en la tenencia de uso del auto particular y las preferencias del modo de transporte que mejor responde a la tipología morfológica de cada sector. 
Densidades, usos y actividades

Los resultados confirman que las densidades más altas están más próximas al centro urbano, donde además hay un mayor grado de ocupación del suelo, con construcciones de altura media. En la distribución de los hogares de los grupos muestrales por sector fue posible observar la mayor dispersión de los participantes de la muestra en los sectores de mayores ingresos, en comparación con los sectores de menores ingresos.

En todos los sectores los rasgos de actividades son predominantemente residenciales; sin embargo, se puede encontrar otros tipos de actividades en los barrios. En efecto, servicios y equipamientos aparecen en mayor medida en los sectores más cercanos al centro urbano, como $\mathrm{BU}$ y LV, distribuidos primordialmente en los bordes del barrio, paralelos a las vías que le dan acceso y conectan con el resto de la ciudad. En menor medida, también se encontraron equipamientos de esta naturaleza en los sectores más lejanos, destacándose equipamientos de tipo comunitario y religioso en ss, como se puede apreciar en el Gráfico 2.

\section{GRÁfICO 2 | Mixtura de usos}

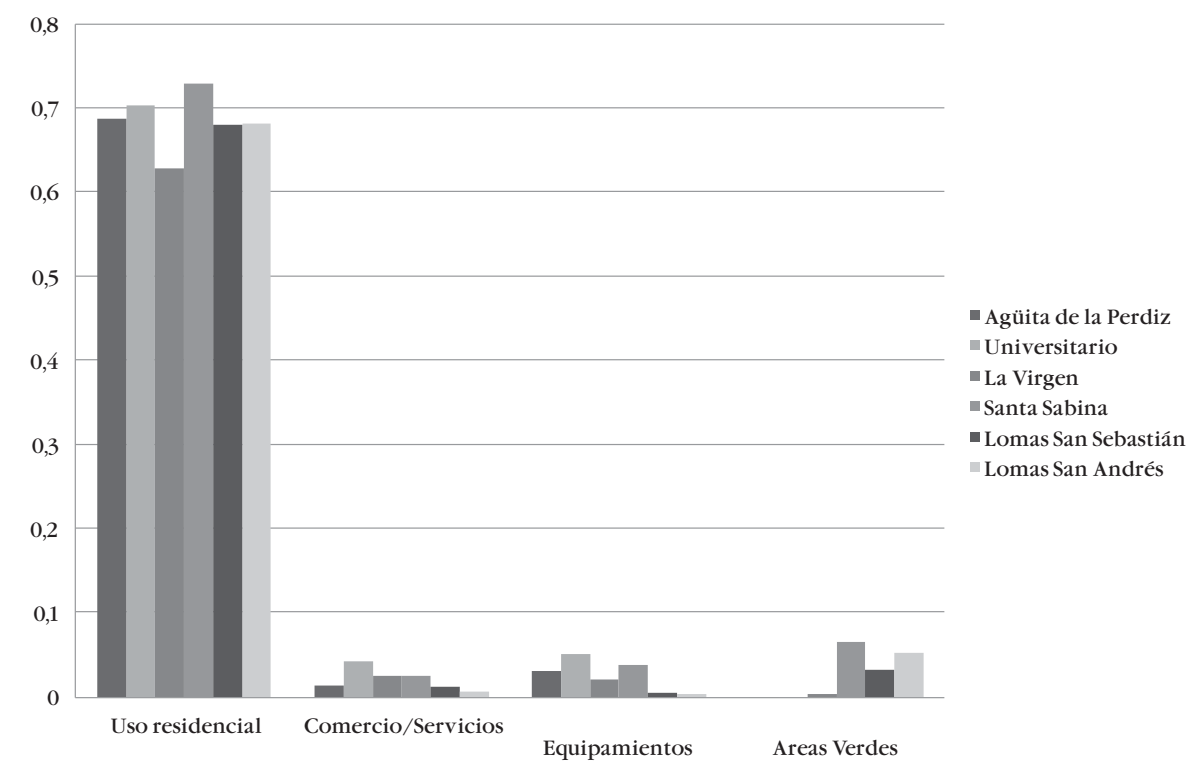

FUENTE ELABORACIÓN PROPIA BASADA EN DATOS RECOLECTADOS

\section{Distribución espacial de las interacciones sociales}

En relación con la localización de los contactos con quienes los entrevistados interactúan con más frecuencia se observa un cierto grado de "localismo" (Marques et al., 2008), especialmente en los sectores de AP y ss (Gráfico 3), con 66,3\% y 51,2\%, respectivamente. En contraste, el porcentaje de contactos que residen en el mismo sector que el entrevistado se reduce en los sectores de ingresos más altos, con 17,1\% en $\operatorname{LV}$ y $22,3 \%$ en LSS. 
GRÁfICO 3| Localización de los contactos - Interacción más frecuente

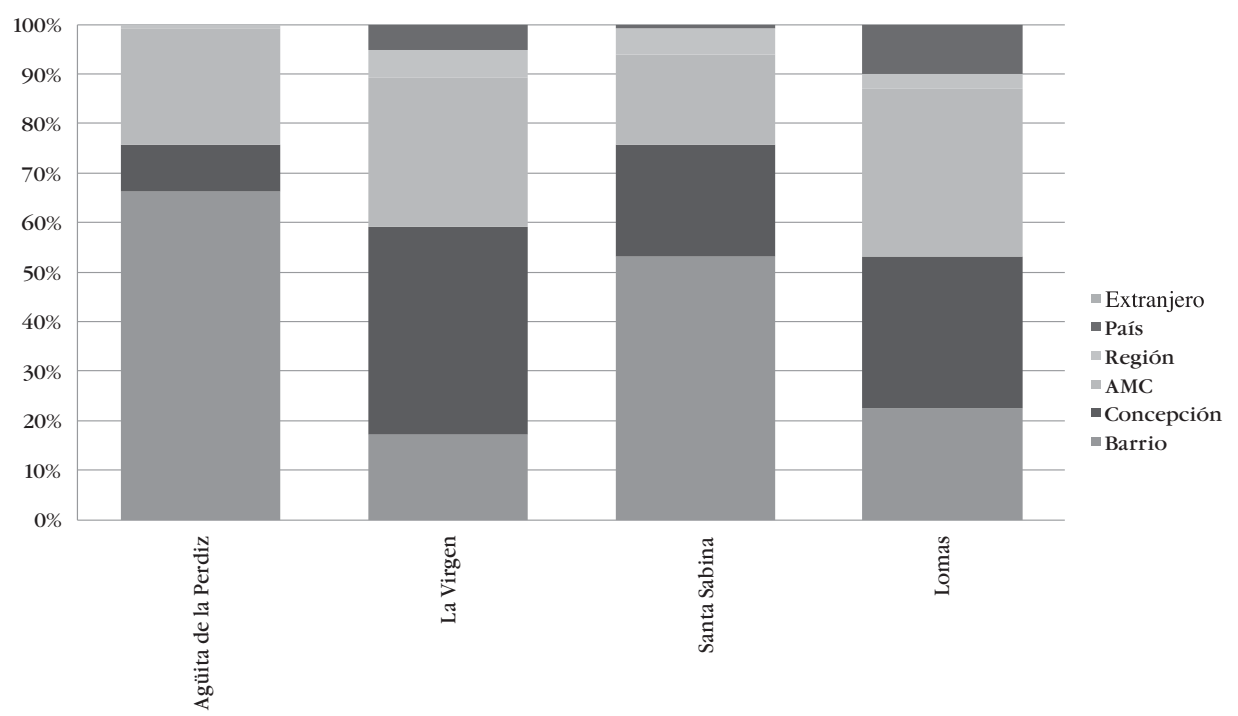

FUENTE ELABORACIÓN PROPIA BASADA EN DATOS RECOLECTADOS

De los contactos con quienes los participantes interactúan con mayor frecuencia, entre $86,1 \%$ y $99,1 \%$, se encuentran al interior del AMC. Los resultados anteriores son consistentes con estudios previos acerca de la relevancia de las relaciones locales en grupos socioeconómicos de ingresos bajos (Holanda, 2000; Marques et al., 2008). Las interacciones frecuentes en los sectores de estratos socioeconómicos más altos se localizan en sectores de la ciudad cuyos estratos socioeconómicos son similares. Fuera del entorno residencial, los puntos de interacción más frecuente se concentran en torno al centro de comercio y servicios de la ciudad, donde hay una mayor densidad de comercio y servicios, además de la mayoría de los lugares de trabajo de los participantes. Cabe destacar que, pese a la cercanía con el sector Trébol -con equipamientos y servicios alternativos al centro comercial tradicional-, no hubo menciones significativas a este sector como lugar de interacción o actividad social.

En los sectores de menor ingreso el fenómeno se repite, siendo posible incluso observar que la mantención de contactos está relacionada con el lugar de origen de los moradores que conformaron inicialmente el barrio. Lo anterior sucede tanto en el caso de AP como de ss, revelando la historia propia de los sectores y la conformación de los tejidos urbanos de estos sectores de recursos bajos.

Ahora bien, indistintamente del sector y grupo socioeconómico, el lugar mayormente citado para las interacciones frecuentes es el hogar, como se puede apreciar en el Gráfico 4. Cabe notar que la categoría hogar incluye tanto el del entrevistado como el de su contacto social. En menor medida, también están presentes en esta categoría los hogares de terceros con los cuales se comparte un vínculo común. En conjunto, los hogares representan entre el 73\% (AP) y el 79,6\% (LSS/LSA) de los lugares de interacción frecuente mencionados en la encuesta. Este indicador expone la importancia de la localización de estos lugares y su distribución geográfica, siendo una parte muy relevante del espacio de actividades de los individuos en el contexto de sus redes personales. 
GRÁfICO 4| Lugar de interacción más frecuente

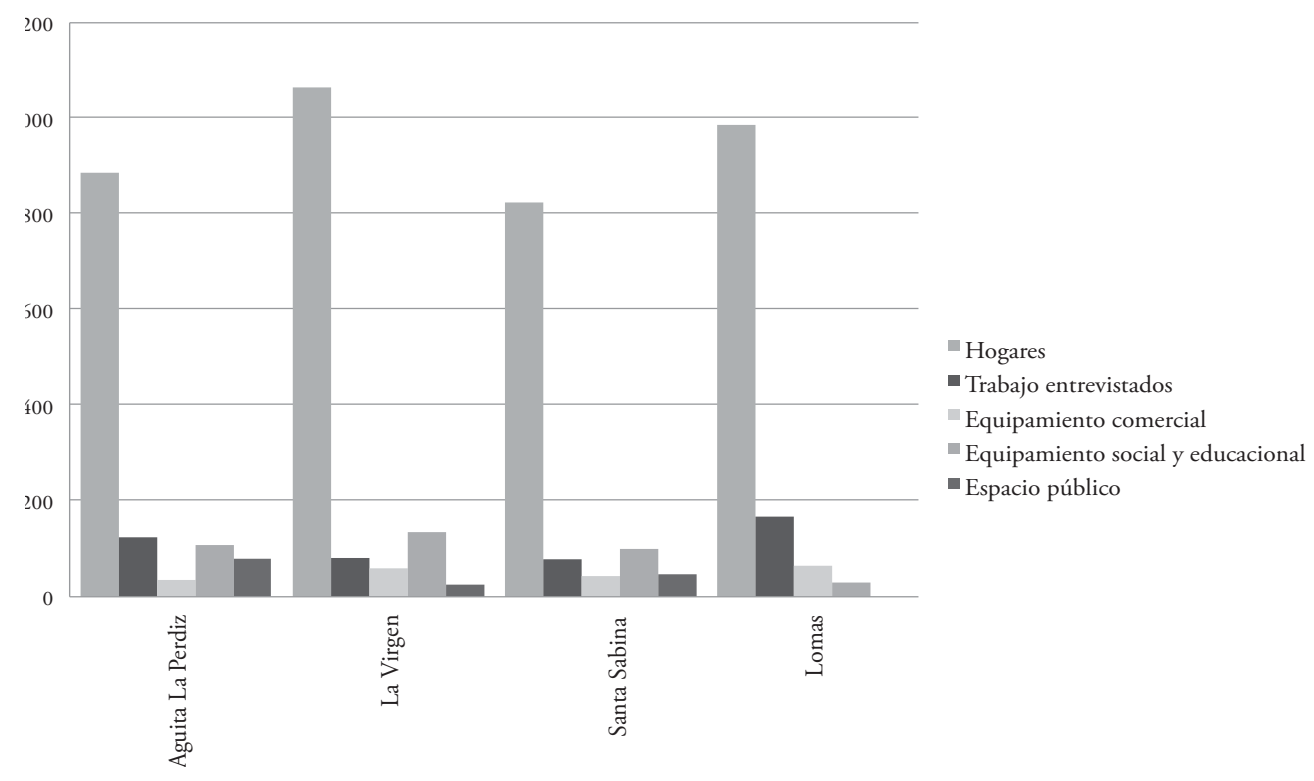

FUENTE ELABORACIÓN PROPIA A PARTIR DE DATOS RECOLECTADOS

Las características generales de la estructura de las redes de cada sector se observan en el Cuadro 4. El tamaño de las redes es estadísticamente equivalente entre los distintos barrios (Bustos, 2011), indicando que la localización y las características socioeconómicas - al menos en lo que se refiere a la muestra en estudio- no revelan incidencia en la cantidad de contactos en las redes. La densidad de la red -indicador que mide su cohesión - es relativamente más alta en los sectores de menores ingresos: 0,27 para AP y 0,26 para ss, comparado a valores de 0,19 y 0,23 en LV y LSs, respectivamente. Estos resultados son coherentes con los tipos de lazos existentes en las redes sociales de estos sectores, cuyos contactos son principalmente familiares o vecinos que viven cerca, y que se conocen entre sí.

CUADRo 4 | Indicadores de estructura de redes personales

\begin{tabular}{|l|c|c|c|c|}
\cline { 2 - 5 } \multicolumn{1}{c|}{} & $\begin{array}{c}\text { AGÜITA DE LA } \\
\text { PERDIZ }\end{array}$ & $\begin{array}{c}\text { BARRIO LA } \\
\text { VIRGEN }\end{array}$ & SANTA SABINA & SAN SEBASTIÁN \\
\hline Tamańo & 21,0 & 23,1 & 18,4 & 21,0 \\
\hline Grado & 5,0 & 3,6 & 3,9 & 4,4 \\
\hline Intermediación & 3,6 & 4,0 & 3,4 & 4,9 \\
\hline Cercanía & 258,1 & 404,5 & 235,8 & 315,1 \\
\hline Densidad & 0,27 & 0,19 & 0,26 & 0,23 \\
\hline Aislados & 1,7 & 3,8 & 2,0 & 2,6 \\
\hline Componentes & 4,8 & 7,3 & 4,8 & 5,4 \\
\hline Cliques & 4,5 & 4,6 & 3,2 & 4,3 \\
\hline Componentes aislados & 3,2 & 3,5 & 2,8 & 2,9 \\
\hline
\end{tabular}

FUENTE ELABORACIÓN PROPIA BASADA EN DATOS RECOLECTADOS 


\section{Trayectos y espacios de actividades}

El análisis del espacio de actividades generado en torno a la red personal, y de las interacciones sociales de los individuos, buscó identificar la existencia de un patrón de comportamiento generado en función de la red de vías y el entorno urbano de los lugares de interacción social, considerando la diversidad y densidad de actividades insertas en ese espacio de actividades.

Pese a que los resultados de los casos de estudio no son del todo concluyentes en identificar patrones de comportamiento de los individuos en relación con el contexto urbano, la visualización de los espacios de actividades fue muy útil cuando fue puesta en paralelo con la estructura de la red social personal del individuo. En ese sentido, y consecuente con Schonfelder y Axhausen (2003), cada individuo desarrolla sus actividades sociales circunscrito a un espacio delimitado, que se genera en función de las interacciones sociales que el individuo tiene con los contactos de su red social. Esto sugiere que el espacio de actividades se construye en el territorio de la ciudad como una suerte de reflejo de la estructura de la red social en la que el individuo está inserto, de modo que estas redes sociales son espacialmente más concentradas y reducidas al territorio "local" de los barrios en el caso de los grupos de menores ingresos, y más dispersas y extendidas en el territorio en los grupos de mayores ingresos.

\section{Discusión}

Si bien el tamaño de las redes sociales no presenta diferencias significativas entre los grupos socioeconómicos, existe un importante grado de "localismo" en las redes de los grupos socioeconómicos menos favorecidos, resultado concordante con investigaciones llevadas a cabo en Brasil (Holanda, 2000; Marques et al., 2008). Los casos en estudio revelan que la localización del trabajo o estudio del participante, en conjunto con la localización de los hogares, tiene un valor gravitante en la definición del espacio de actividades. La densidad y la diversidad de actividades en el contexto urbano cercano al trabajo también posibilitan las interacciones sociales con miembros de la red social fuera del ámbito laboral y en espacios fuera del hogar (Ronald \& Arentze, 2009). Este resultado puede explicar la casi inexistente mención del sector El Trébol como lugar de interacción y actividad social, ya que la gran concentración de los lugares de trabajo de la muestra general está en el centro de comercio y servicios tradicional, reforzando este carácter de centralidad gravitante en el AMC.

El "localismo" presente sugiere la relevancia del barrio como lugar donde se produce la mayoría de las interacciones sociales de los estratos socioeconómicos más bajos. Al mismo tiempo, en estos barrios, equipamientos que propician la socialización, como asociaciones culturales, sociales y religiosas -en los que la participación no necesariamente implica inversión de recursos económicos- tienen cierta importancia cuando el lugar de interacción frecuente es fuera del hogar.

La localización cercana al centro de servicios de AP favorece la posibilidad de acceder a las actividades sociales en un contexto urbano más diverso, considerando las restricciones presupuestarias que predominan para la realización de tales interacciones. De esta forma, al estar cerca del centro es posible acceder a una actividad social a pie, y el dinero que se invertiría en transporte puede dirigirse a compartir 
con amigos en un lugar comercial. Esta situación es más restrictiva en ss, ya que el sector se encuentra alejado del centro de servicios, pese a contar con buena oferta de transporte público.

Los datos empíricos muestran que, indistintamente del grupo socioeconómico de que se trate, los hogares son los lugares de interacción más mencionados en la muestra. Pese a esto, el hogar está reservado preferentemente para familiares y amigos, apareciendo en menor medida para los compañeros de trabajo, estudio y organizaciones sociales. Lo anterior puede relacionarse con una diferenciación de las interacciones y actividades sociales entre una esfera pública y una privada, y en función del rol que los participantes de una actividad tienen en la estructura de la red social. En los grupos de ingresos más bajos, los integrantes del círculo de contactos emocionalmente cercanos, en general, también son muy cercanos en cuanto a distancia entre hogares; esto se puede explicar por la presencia de vecinos en las redes personales de estos grupos, y la tendencia a permanecer en el barrio donde están sus familiares. Por otra parte, en los grupos de altos ingresos el grado de cercanía en el círculo de contactos de la red no está relacionado con la cercanía física, porque este grupo tiene más recursos para mantener activo este contacto por otros medios, más allá de la interacción cara a cara.

En los sectores de AP y ss la configuración de la vialidad y la estructura de la red favorecen en mayor medida el modo caminata y transporte público, aunque con matices, si se considera la calidad de la infraestructura de veredas y urbanización. A lo anterior se suma que la condición topográfica de ambos sectores no fue considerada en la conformación de los asentamientos, generando espacios con accesos restringidos y con pronunciadas pendientes, lo que limita la accesibilidad de personas mayores, así como la oferta de facilidades para realizar actividades sociales.

Las interacciones sociales en los barrios de menores ingresos se restringen, en la mayor parte de los caso, al interior del sector, entre hogares que se sitúan con la cercanía suficiente para el recorrido peatonal, lo que es uno de los generadores del sentido de arraigo e identificación con el lugar a lo largo del tiempo y, por lo tanto, responsable del enorme sentido de apropiación espacial que allí se da (Grande, 2007; Vidal \& Pol, 2005). Sin embargo, esta condición de identificación con el lugar, positiva en términos sociales (Gustafson, 2001), también puede reforzar la condición de exclusión y segregación residencial.

En los barrios de mayores ingresos, en cambio, en general no hay restricciones en cuanto a la distancia para las interacciones sociales, con viajes más largos y mayoritariamente recorridos en automóvil. En este caso, el vínculo con el contexto urbano en que se produce la experiencia urbana está circunscrito a la velocidad de viaje del auto, por las vías percibidas como los accesos de lugar a lugar más directos o más rápidos (Wellman, 2001; 2005). De esta forma, la relación con el entorno en términos de apreciación de los espacios se da, de forma simbólica, como una valoración del paisaje recorrido más que por el "vivido" (Holanda, 2000).

Esta situación es mucho más evidente en el sector de LSs, pese a que el planteamiento del espacio cerrado (condominio) y el control de acceso sea un discurso inicial válido para justificar la seguridad y el uso del espacio solo por "conocidos". Sin embargo, se observa que, en estos espacios, la interacción con vecinos es casi 
inexistente, y ocurre primordialmente por situaciones funcionales y ocasionales. De esta forma, el espacio de fricción planteado por Grannis $(1998 ; 2005)$ en los sectores de altos ingresos no se produce, pese a la existencia de buena infraestructura de veredas y áreas verdes equipadas, ya que la morfología urbana promueve y favorece el uso del auto particular prácticamente de puerta a puerta entre hogares, los que generalmente están distantes entre sí y no pertenecen al mismo sector. Aún más, las propiedades del sistema vial que componen la red del sector de Lss indican una integración local muy baja, lo que se suma a una menor diversidad de equipamientos, aspectos relevantes para promover la caminata, modo esencial para que se produzca el encuentro entre vecinos, como ocurre en ss y AP. De este modo, las redes sociales primarias de los contactos cercanos en los sectores de mayor ingreso tienden a estar mucho más relacionadas con amigos y familiares que con vecinos, toda vez que el contexto urbano de estos lugares no favorece el encuentro entre ellos.

Respecto de los contactos sociales, los estratos socioeconómicos de menores ingresos siguen vinculados a sus redes a partir del espacio físico, por cercanía y por el contacto diario en la calle. De este modo es posible encontrar en estos sectores un vínculo afectivo con el entorno mucho más arraigado que en los sectores de altos ingresos, lo que además es reforzado por el hecho de que parte de la red personal afectiva y familiar también vive en las cercanías físicas. Por estas mismas razones, el espacio de actividades por donde se mueven e interactúan es más concentrado, usualmente con una base muy marcada al interior de los propios sectores. Esta situación genera una suerte de autosegregación residencial y autoexclusión social, no necesariamente explícita, pero que se agudiza si el sector está alejado del centro de servicios, como ocurre con ss.

En el caso de los grupos socioeconómicos de ingresos más altos, estos tienen la posibilidad de diferenciar sus espacios de interacción. En otras palabras, sus espacios de actividades no están vinculados directamente con el sector donde viven, ya que sus redes personales tampoco lo están, lo que implica actividades más dispersas en la ciudad, favorecidas por el uso del vehículo particular. Además, el contexto urbano de estos sectores ofrece mayor integración global con los lugares donde están ubicados sus contactos y se realizan sus actividades sociales. En general, los sectores de ingresos medio-altos están muy bien conectados con otros sectores de la ciudad, por medio de vías estructurantes o expresas. De este modo, el vínculo que se produce con el entorno urbano es débil; está relacionado únicamente con el tránsito de un punto geográfico específico a otro, en su mayoría hogares de los actores, y representa una relación mucho más situacional que de arraigo. De esta forma, ambos grupos sociales -altos y medio-altos- establecen un vínculo de experiencia urbana reducida frente al universo global de la ciudad, con importantes diferencias en cuanto a su relación con ella.

Los grupos sociales de menores ingresos están más integrados localmente, con un arraigo mucho mayor al interior de sus barrios, pero con una reducida integración a nivel global. Lo anterior ocurre no solo por restricciones económicas, sino también condicionado por la estructura de la red social en la cual están insertos, ya que los integrantes de sus redes sociales con quienes interactúan regularmente también están ubicados en el mismo barrio. Aún más, el contexto urbano, considerando la 
estructura de la red vial de estos entornos, refuerza tal tendencia. Por otro lado, los grupos de mayores ingresos establecen un vínculo de lugar a lugar o persona a persona (Wellman, 2001), permitido por la libertad del auto particular, lo que genera una integración con la ciudad más global. Sin embargo, estos grupos poseen una débil integración local, con escaso vínculo afectivo y hasta desconocimiento del sector donde viven (Greene \& Mora, 2005).

Como consecuencia, la mirada desde la perspectiva de las redes sociales revela una nueva arista de la segregación socioespacial como fenómeno de transformación urbana, resultado del crecimiento y metropolización del AMC (Hidalgo, 1999; Machiavello \& Hidalgo, 2010; Rojas et al., 2009). El enfoque de ARs muestra que los fenómenos de exclusión y segregación social que se dan en el espacio de la ciudad son, en parte, un reflejo de las interacciones sociales de los individuos en el contexto de sus redes sociales, y del entorno urbano donde se desarrollan. De esta forma, la proximidad física entre grupos sociales de marcadas diferencias sociales, posible de encontrar en ciudades chilenas (Sabatini \& Brain, 2008) como producto del proceso de metropolización, parece no ser suficientemente significativa para generar espacios de integración y mayor capital social entre los distintos grupos socioeconómicos. Esto indica que otros aspectos deben ser incorporados al análisis del contexto urbano para entender su relación con las interacciones sociales.

En esa línea, la revelación de los hogares como centro focal de las interacciones sociales, de manera transversal a la condición socioeconómica, indica que la constitución de los asentamientos residenciales y su localización en relación con la accesibilidad a las facilidades de servicios, comercio, equipamiento, trabajo, entre otros, que ofrecen los centros urbanos, es un tema muy relevante en la construcción social y espacial de la ciudad. Esto sugiere la necesidad de intervención de políticas públicas que apunten a aumentar la integración global de los sectores de menores ingresos y la integración local de los sectores de mayores ingresos. De esta manera, se podría valorizar y generar espacios en el contexto urbano que propicien la sobreposición y posibilidad de co-presencia de grupos sociales más diversos.

Los espacios de actividades de los distintos grupos socioeconómicos, fruto de sus redes sociales, se generan a partir de distintas capas que se confrontan en una suerte de dualidad sobre el territorio de la ciudad. Estas capas se traslapan en pocas oportunidades, impidiendo las condiciones de fricción que, según plantea Grannis (2009), son necesarias para que la interacción social entre distintos grupos sociales se produzca. Lo anterior lleva a la discusión preconizada por Jacobs (1961), quien arguye que la fragmentación urbana, tanto social como espacial, es un elemento clave en la producción de exclusión y segregación social. La poca relevancia del espacio público como espacio de interacción social, apreciada en este estudio, abre una inquietud acerca de su rol en cuanto promotor de interacción social. Finalmente, es preocupante la debilidad del espacio público apreciada en este estudio, considerando que las relaciones sociales y las actividades humanas, concebidas como comunidad de redes, definen y justifican la existencia de las ciudades y son un espejo de ellas. 


\section{Agradecimientos}

Los autores agradecen el financiamiento de la Comisión Nacional de Investigación Científica y Tecnológica (Conicyt), por medio del Fondo Nacional de Desarrollo Científico y Tecnológico (Fondecyt), Proyecto $\mathrm{N}^{\circ} 1110920$, y el Programa de Financiamiento Basal para Centros de Excelencia, Instituto Sistemas Complejos de Ingeniería, FBO-16. También quieren expresar su reconocimiento a Pamela Valdebenito, por su colaboración en el procesamiento de los casos de estudio.

\section{Referencias bibliográficas}

Agurto, L. (2002). Aproximación al proceso de apropiación del espacio colectivo en asentamientos espontáneos urbanos de Concepción. Concepción: Carrera de Arquitectura, Facultad de Arquitectura Universidad de Concepcion.

Atria, R., Siles, M., Arriagada, I., Robinson, L. \& Whiteford, S. (Comps.). (2003). Capital social y reducción de la pobreza en América Latina y el Caribe: en busca de un nuevo paradigma. Santiago de Chile: Comisión Económica para América Latina y el Caribe (CEPAL) / Universidad del Estado de Michigan (MSU). En http://goo.gl/k7m5JM

Axhausen, K. W. (2006). Social networks, mobility biographies and travel: The survey challenges. Arbeitsbericht Verkehrs- und Raumplanung, 343. En http://e-collection. library.ethz.ch/eserv/eth:28874/eth-28874-01.pdf

Boarnet, M. \& Crane, R. (2001). The influence of land use on travel behavior: specification and estimation strategies. Transportation Research Part A: Policy and Practice, 35(9), 823-845. http://dx.doi.org/10.1016/S0965-8564(00)00019-7

Bustos, C. (2011). Formulación y análisis de indicadores del espacio de actividades de individuos del Gran Concepción: Caso de las actividades sociales. Concepción: Universidad de Concepción.

Cao, X., Mokhtarian, P. L. \& Handy, S. L. (2007). Do changes in neighborhood characteristics lead to changes in travel behavior? A structural equations modeling approach. Transportation, 34(5), 535-556. En http://escholarship.org/uc/item/49393360\#page-2

Carrasco, J. A., Bustos, C. \& Cid-Aguayo, B. (2013). Affective personal networks versus daily contacts: Analyzing different name generators in an social activity-travel behaviour context. En J. Zmud, M. Lee-Gosselin, M. Munizaga \& J .A. Carrasco (Eds.), Transport Survey Methods: Best Practice for Decision Making (pp. 409-426). London: Emerald Press.

Carrasco, J. A. \& Miller, E. J. (2006). Exploring the propensity to perform social activities: a social network approach. Transportation, 33(5), 463-480. doi: 10.1007/s11116-006-8074-z

Carrasco, J. A, \& Miller, E. J. (2009). The social dimension in action: A multilevel, personal networks model of social activity frequency between individuals. Transportation Research Part A: Policy and Practice, 43(1), 90-104. doi:10.1016/j.tra.2008.06.006

Carrasco, J. A., Hogan, B., Wellman, B. \& Miller, E. J. (2008). Collecting social network data to study social activity-travel behavior: an egocentric approach. Environment and Planning B: Planning and Design, 35(6), 961-980. doi: 10.1068/b3317t 
Cervero, R. (2003). The built environment and travel : Evidence from the United States. European Journal of Transport and Infrastructure Research, 3(2), 119-137. En http:// onlinepubs.trb.org/onlinepubs/sr/sr282.pdf

Crane, R. (2000). The Influence of urban form on travel: An interpretive review. Journal of Planning Literature, 15(1), 3-23. doi: 10.1177/08854120022092890

Ewing, R. \& Cervero, R. (2010). Travel and the built environment: a meta analysis. Journal of the American Planning Association, 76(3), 265-294. doi: 10.1080/01944361003766766

Ewing, R., Handy, S., Brownson, R. C., Clemente, O. \& Winston, E. (2006). Identifying and measuring urban design qualities related to walkability. Journal of Physical Activity and Health, 3(Supl. 1), 223-239. En http://activelivingresearch.org/sites/default/files/ JPAH_15_Ewing.pdf

Fan, Y. \& Khattak, A. J. (2009). Does urban form matter in solo and joint activity engagement? Landscape and Urban Planning, 92(3-4), 199-209. http://dx.doi.org/10.1016/j. landurbplan.2009.05.006

Grande, P. E. De. (2007). Redes personales y espacio urbano. En V Mesa Hispana para el análisis de redes sociales. Methodology of social networks within social research. XXVII International Sunbelt Social Network Conference. Corfú, Grecia, 1 de mayo de 2007. En http:// revista-redes.rediris.es/webredes/vmesahispana/espaciou.pdf

Grannis, R. (1998). The importance of trivial streets: Residential streets and residential segregation 1. American Journal of Sociology, 103(6), 1530-1564.

Grannis, R. (2005). T-Communities: Pedestrian street networks and residential segregation in Chicago, Los Angeles, and New York. City \& Community, 4(3), 295-322. doi: 10.1111/j.1540-6040.2005.00118.x

Grannis, R. (2009). From the ground up: Translating geography into community through neighbor networks. Princeton, NJ: Princeton University Press.

Greene, M. \& Mora, R. (2005). Las autopistas urbanas concesionadas: Una nueva forma de segregación. ARQ (Santiago), (60), 56-58. http://dx.doi.org/10.4067/S071769962005006000009

Gustafson, P. (2001). Meanings of place: Everyday experience and theoretical conceptualizations. Journal of Environmental Psychology, 21(1), 5-16. http://dx.doi.org/10.1006/ jevp.2000.0185

Handy, S., Cao, X. \& Mokhtarian, P. (2005). Correlation or causality between the built environment and travel behavior? Evidence from Northern California. Transportation Research Part D: Transport and Environment, 10(6), 427-444. http://dx.doi. org/10.1016/j.trd.2005.05.002

Hidalgo, R. (1999). Continuidad y cambio en un siglo de vivienda social en Chile (18921998). Reflexiones a partir del caso de la ciudad de Santiago. Revista de Geografía Norte Grande, 26, 69-77. En http://www.geo.puc.cl/laburb/investigacion/vivienda/pdf\%20 articulos/NG\%20-\%2026.pdf

Hillier, B. (1996). Space is the machine: a configurational theory of architecture. London: Space Syntax. En http://www.ninsight.at/ak_stdb/SpaceIsTheMachine.pdf

Hillier, B. \& Hanson, J. (1984). The social logic of space. [Cambridge Books Online: 2009, dic.]. Cambridge: Cambridge University Press. http://dx.doi.org/10.1017/ CBO9780511597237 
Holanda, F. de. (2000). Class footprints in the landscape. Urban Design International, 5, 189198. doi:10.1057/palgrave.udi.9000015

Jacobs, J. (1961). The death and life of great American cities. Nueva York: Random House.

Jones, P. (2009). The role of an evolving paradigm in shaping international transport research and policy agendas over the last 50 years. Proceedings of the XII International Association for Travel Behaviour Research Conference (iatbr2009.asu.edu). En R. M. Pendyala \& Ch. R. Bhat (Eds.), Travel behaviour research in an evolving world (3-34). Lulu Publishers (USA).

Kitamura, R., Akiyama, T., Yamamoto, T. \& Golob, T. (2001). Accessibility in a metropolis: Toward a better understanding of land use and travel. Transportation Research Record, 1780(1), 64-75. http://dx.doi.org/10.3141/1780-08

Kitamura, R. \& Mokhtarian, P. (1997). A micro-analysis of land use and travel in five neighborhoods in the San Francisco Bay Area. Transportation, 24(2), 125-158. doi:10.1023/A:1017959825565

Lin, N. (2005). A network theory of social capital. En D. Castiglione, J. van Deth \& G.Wolleb (Eds.), Handbook on Social Capital (pp. 1-25). Oxford, Uk: Oxford University Press. Artículo disponible en http://www.pro-classic.com/ethnicgv/SN/SC/paperfinal-041605.pdf

Lozares, C. (1996). La teoria de redes sociales. Papers, 48, 103-126. En http://alejandrogg.com. $\mathrm{mx} /$ temario3/Carlos-redes\%20sociales.pdf

Machiavello, F. \& Hidalgo, R. (2010). Consecuencias socioespaciales de los conjuntos de vivienda social en el Área Metropolitana de Concepción (AMC): ¿Barrios integrados o espacios urbanizados? En L. Pérez \& R. Hidalgo, Concepción Metropolitano. Evolución $y$ desafíos (pp. 153-170). Concepción: Editorial Universidad de Concepción / Instituto de Geografía, Pontificia Universidad Católica de Chile, Serie GEOlibros.

Marques, E., Moya, E. \& Zoppi, M. (2008). Personal networks and urban poverty: Preliminary findings. Brazilian Political Science Review (Online), 3, 10-34. En http://socialsciences. scielo.org/pdf/s_bpsr/v3nse/a01v3nse.pdf

Marshall, S. (2005). Streets Patterns. London: Spon Press.

McCarty, C. (2002). Structure in personal networks. Journal of Social Structure, 3, 1-29. En http://www.cmu.edu/joss/content/articles/volume3/McCarty.html

McPherson, M., Smith-Lovin, L., \& Cook, J. M. (2001). Birds of a feather : Homophily in social networks. Annual Review of Sociology, 27(I), 4I 5-444. doi: 10.1146/annurev. soc.27.1.41

Mok, D., Wellman, B., \& Carrasco, J. (2010). Does distance matter in the age of the internet? Urban Studies, 47(13), 2747-2783. doi: 10.1177/0042098010377363

Mokhtarian, P. L. \& Cao, X. (2007). Examining the impacts of residential self-selection on travel behavior: A focus on methodologies. Transportation Research Part B: Methodological, 42(3), 204-228. http://dx.doi.org/10.1016/j.trb.2007.07.006

Næss, P. (2004). Urban structures and travel behaviour. Experiences from empirical research in Norway and Denmark. European Journal of Transport and Infrastructure Research, 3(2), 155-178. En http://www.ejtir.tbm.tudelft.nl/issues/2003_02/ abstracts/2003_02_03.asp

Næss, P. (2005). Residential location affects travel behavior: but how and why? The case of Copenhagen metropolitan area. Progress in Planning, 63(2), 167-257. http://dx.doi. org/10.1016/j.progress.2004.07.004 
Netto, V. de M. \& Krafta, R. (2001). Socio-spatial networks. Social segregation as a realtime phenomenon. Proceedings of the III International Space Syntax Symposium, Atlanta 2001. Ponencia disponible en http://www.ucl.ac.uk/bartlett/3sss/papers_pdf/34_ netto\&krafta.pdf

Panerai, P. (2006). Analise urbana. Brasilia: Editora UnB.

Pérez, L. \& Salinas, E. (2009). Fragmentos de ciudad. Formas de crecimiento del Concepción Metropolitano. En R. Hidalgo, C. De Mattos \& F. Arenas (Eds.), Chile: del país urbano al país metropolitano (pp. 269-300). Santiago de Chile: Ediciones Universidad Católica de Chile, Serie GeoLibros.

Rojas, C., Muñiz, I. \& García_López, M. Á. (2009). Estructura urbana y policentrismo en el Área Metropolitana de Concepción. EURE, 35(105), 47-70. http://dx.doi. org/10.4067/S0250-71612009000200003

Rojas, C., Opazo, S. \& Jaque, E. (2009). Dinámica y patrones de crecimiento del Área Metropolitana de Concepción. Tendencias de las últimas décadas. En R. Hidalgo, C. De Mattos \& F. Arenas (Eds.), Chile: del país urbano al país metropolitano (pp. $257-$ 268). Santiago de Chile: Ediciones Universidad Católica de Chile, Serie GeoLibros.

Ronald, N. \& Arentze, T. (2009). An agent-based framework for modelling social influence on travel behaviour. En R. S. Anderssen, R. D. Braddock \& L. T. H Newham (Eds.), Proceedings of the 18th World IMACS Congress and MODSIM09 International Congress on Modelling and Simulation (pp. 2955-2961). Cairns, Australia,

Sabatini, F. \& Brain, I. (2008). La segregación, los guetos y la integración social. EURE, 34(103), 5-26. http://dx.doi.org/10.4067/S0250-71612008000300001

Schonfelder, S. \& Axhausen, K. W. (2003). Activity spaces : measures of social exclusion? Transport Policy, 10(4), 273-286. http://dx.doi.org/10.1016/j.tranpol.2003.07.002

Solá-Morales, M. de. (1996). Las formas de crecimiento urbano. Barcelona: Edicions Universitat Politècnica de Catalunya (UPC).

Stead, D. \& Marshall, S. (2001). The relationships between urban form and travel patterns. An international review and evaluation. European Journal of Transport and Infrastructure Research, 1(2), 113-141. En http://www.ejtir.tbm.tudelft.nl/issues/2001_02/ abstracts/2001_02_01.asp

Vidal, T. \& Pol, E. (2005). La apropiación del espacio : una propuesta teórica para comprender la vinculación entre las personas y los lugares. Journal of Environmental Psychology, 36(1), 281-297. En http://www.redalyc.org/articulo.oa?id=97017406003

Wellman, B. (1997). Structural analysis : from method and metaphor to theory and substance. En B. Wellman \& S. D. Berkowitz (Eds.), Social Structures. A network approach (pp. 19-61). Cambridge: Cambridge University Press. Artículo disponible en http:// homepage.ntu.edu.tw/-khsu/network/reading/wellman2.pdf

Wellman, B. (2001). Physical place and cyberplace: The rise of personalized networking. International Journal of Urban and Regional Research, 25(2), 227-252. doi: 10.1111/1468-2427.00309

Wellman, B. (2005). Community: from neighborhood to network. Communications of the $A C M, 48(10), 53-55$. doi: 10.1145/1089107.1089137 